\title{
DIMORPHISM IN THE SPERMATOZOA OF NECTURUS MACULOSUS
}

\author{
HELEN DEAN KING
}

From The Wistar Institute of Anatomy and Biology

SIX FIGURES

A study of the mature spermatocytes in the testes of Necturus maculosus has shown that these cells contain a peculiar multiple chromosome that divides unequally in the first maturation mitosis and thus leads to the formation of two classes of spermatozoa, one having more chromatin than the other. As a dimorphism in the spermatozoa of amphibians has not heretofore been reported, a brief description of the spermatocyte divisions in Necturus is given in the present paper: a more detailed account will appear when my investigation of the early growth stages of the spermatocytes has been completed.

In a very early prophase of division the primary spermatocytes contain a thick, apparently continuous spireme which is split longitudinally throughout its entire length. This spireme breaks into twelve segments of different lengths which condense rapidly to form the chromosomes for the first maturation spindle.

Two of the chromatin segments of the prophase are distinguished from the others on account of their greater length. One of these segments becomes a very large ring-shaped chromosome which is very conspicuous in the metaphase of the first mitosis when it is usually twisted in various ways (figs. $2, L ; 3, L$ ). In mitosis this chromosome divides equally, forming two $V$-shaped chromosomes which move to opposite poles of the spindle. The other long segment, as its later history shows, is not a bivalent but a multiple chromosome. In. the late prophase this segment appears as a long, thick rod of nearly uniform diameter with the 405

THE ANATOMICAL RECORD, VOL. 6, No. 10 
two ends bent at nearly right angles to the main axis (fig. 1). The bent terminal sections are never of equal length, and the longer one is the 'accessory' or the ' $X$ chromosome' which became attached to one of the large bivalent chromosomes at an early period in the growth of the spermatocytes. Not infrequently, as shown in figure $1, X$, the $X$ chromosomes is detached from the main structure and connected with it only by linin.

The multiple chromosome goes into the first maturation spindle in practically the same form in which it appears in the late prophase, and in the metaphase it assumes such a position on the spindle that its main axis lies along one of the spindle fibers while the two bent terminal portions either project at right angles to the spindle (fig. 2), or they lie across the spindle fibers (fig. 3). There is more or less variation in the size of this chromosome in different spindles, due doubtless to fixation and staining, but in nearly all cases it extends over considerably more than half of the length of the spindle and in some cells it reaches from pole to pole. Sometimes this structure is of nearly uniform thickness throughout its entire length (fig. 3): more frequently, as shown in figure 2 , it has slight constrictions that divide it into five parts and thus give it the appearance of a pentad structure. In its constricted form this chromosome appears remarkably like the multiple chromosome found by McClung ('05) in the spermatocytes of Hesperotettix speciosus and of various other species of orthoptera.

Whenever the multiple chromosome is shown in its entirety the portion of it which forms the $X$ chromosome can readily be distinguished. Sometimes the $X$ component seems to be an integral part of the multiple chromosome, although it invariably forms a sharp angle with the main axis of this structure (fig. 2, $X$ ). At other times, as shown in figure 3 , the $X$ chromosome is a short, rod shaped body lying close against one end of a large bivalent chromosome, but apparently not connected with it in any way.

The bent portion of the multiple chromosome at the end opposite to the $X$ chromosome is usually much shorter than the $X$ component (figs. 2 and 3 ), and as yet $I$ have not found a single case in which this section was not directly continuous with the main axis of the multiple chromosome although always forming an angle 
with it. It seems probable, from the evidence in hand at present, that this bent terminal portion is merely the end of one of the

All figures'were drewn with the aid of a camera lucida under a magnification of about 1500 diameters: they have been reduced one-half. $L$, large ring-shaped chromosome; $S$, supernumerary chromosomes; $X, X$ chromosome.
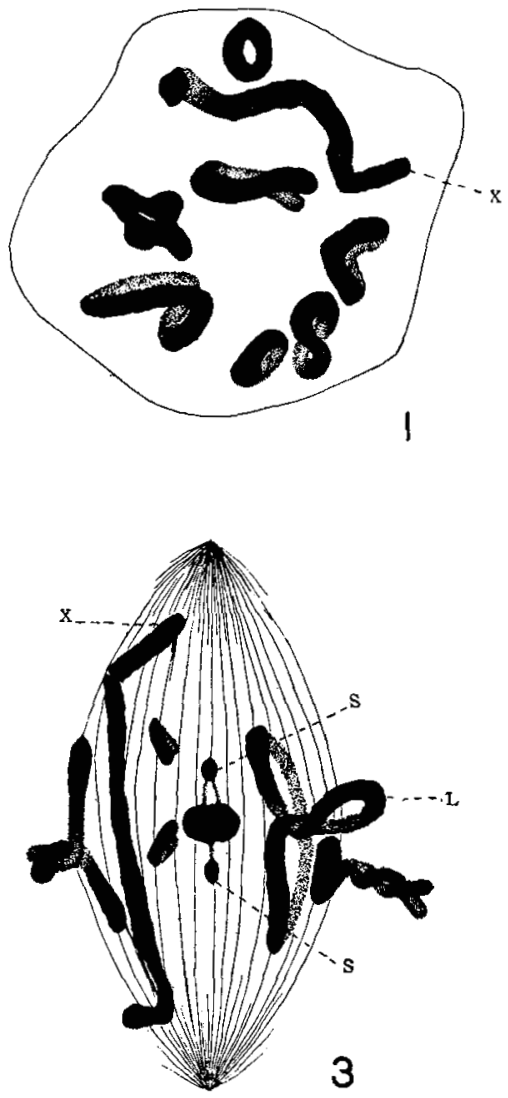

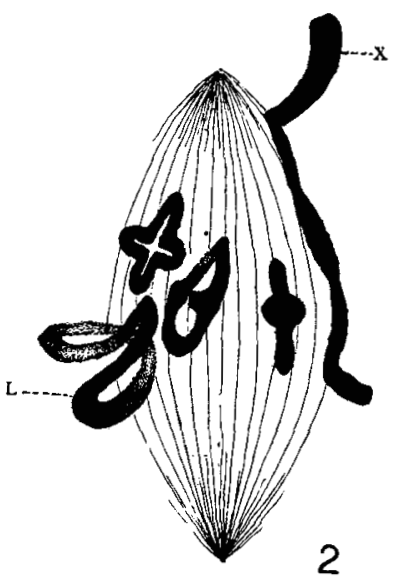

2

Fig. 1 Late prophase of the first maturation mitosis. Figs. 24 Metaphases of the first maturation mitosis.

univalent chromosomes which helped to form the multiple chromosome during an early period in the development of the spermatocytes. There is the possibility, however, that this section is the small mate of the $X$ chromosome, in which case the spermato- 
cytes of Necturus contain an unequal pair of heterochromosomes instead of an. $X$ chromosome. This point cannot be cleared up until the early history of the spermatocytes has been. studied.

When the first maturation mitosis occurs the multiple chromosome divides in such a way that one daughter cell gets one univalent chromosome and the $X$ component (fig. 4) while the rest of the complex goes into the other cell.

The other ten segments of the prophase are considerably shorter than the two described above. At least six of these segments appear as small rings in the late prophase (fig. 1) and go in.to the spindle in this form. All of the remaining segments condense as crosses with one pair of arms considerably longer than the other (figs. 1 and 2). The cross-shaped chromosomes undergo considerable modification in form before the maturation division occurs. At first the longer axis of each of these chromosomes lies along one of the spindle fibers (fig. 2). This axis shortens gradually and its chromatin substance goes into the side arms which increase correspondingly in length. Just before division these chromosomes appear either as short, thick rods which lie across the spindle fibers, or as small elongated rings in which the central opening is a mere slit (fig. 3). In mitosis each of these chromosomes divides through its longitudinal axis, forming two small $V$-shaped chromosomes of equal size. A similar change in the shape of crossshaped chromosomes preparatory to division has been found in the germ-cells of various other species of amphibians (Carnoy and Lebrun '99; Lebrun '01; King '05).

In connection with the change in the shape of the cross-shaped chromosomes in the spermatocytes of Necturus there sometimes occurs a process which, as far as I am aware, has not been observed to take place in the germ-cells of other amphibians in which chromosomes of a similar shape are found. A small mass of chromatin., at one or at both ends of the arms lying along the spindle fiber, becomes so firmly attached to the spindle fiber that it gradually becomes separated from the main body as the side arms increase in size. For a time such small fragments remain attached to the rest of the chromosome by linin-like strands (fig. 3), but eventually they break away from the parent mass and appear on the 
spindle as small, round supernumerary chromosomes (fig. $4, S$ ). These supernumeraries move with the $V$-shaped chromosomes to the spindle poles, and they can sometimes be distinguished in a late anaphase. These small chromatin masses are seemingly derived only from the chromosomes that appear on the spindle in the form of a cross, and as they move to the same pole of the spindle as do the univalent chromosomes to which they belong, their detachment from the main mass of chromatin does not lead to an unequal distribution of chromatin to the spindle poles. Supernumerary chromosomes are not found on all spindles and their formation is apparently due solely to the fact that at times the connection between the ends of the cross-shaped chromosomes and the spindle fibers is stronger than the force that is changing the shape of the chromosome and causing the chromatin to move into the side arms. I have not as yet been able to trace these small chromosomes beyond the first maturation spindle. Small supernumerary chromosomes have been found in the spermatocytes of various species of insects by Stevens (' $12 \mathrm{a}$, ' $12 \mathrm{~b}$ ) and by Wilson. ('09), but they differ from the supernumerary chromosomes of Necturus in that they appear to be derived from the heterochromosomes and not from the ordinary bivalents.

The first maturation mitosis in Necturus is probably a segregation division, and there is seemingly a definite order in which the various chromosomes divide. The multiple chromosome divides after the division of one or of two of the smallest ring-shaped chromosomes (fig. 4); then follows the separation of the univalents which were united as crosses or as rings of medium size; lastly the large ring-shaped chromosome separates into its univalent components. Since the multiple chromosome divides at a very early period it is evident that the order in which the chromosomes divide is not dependent on the size of the chromosomes but on some other factor as yet undetermined.

There is a resting period of considerable length between the two maturation divisions, during which the chromosomes lose their visible identity and form a continuous spireme. The chromosomes that emerge from the spireme to go into the second maturation. spindle are $V$-shaped structures that vary consider- 
ably in size. As a rule two of these chromosomes are considerably larger than the others. One, with arms of equal length, is doubtless the univalent chromosome which with its homologue formed the large ring-shaped chromosome of the first maturation spindle. The other large $V$-shaped chromosome has arms that are slightly unequal. This is probably the chromosome derived from the multiple chromosome of the first spindle. Since the chromosomes are very much crowded together in the second spindle it becomes very difficult to follow the $X$ chromosome. In most cases the $X$ chromosome probably remains attached to one of the univalent chromosomes and divides longitudinally with it
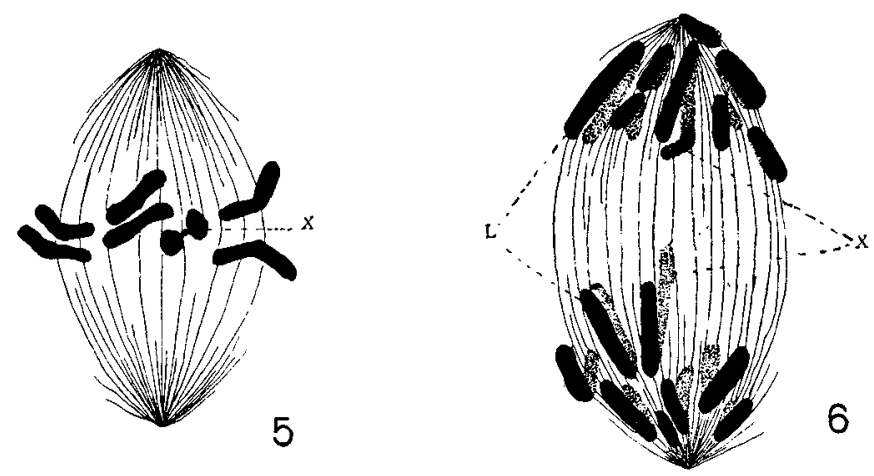

Fig. 5 Early anaphase of the second maturation mitosis.

Fig. 6 Late anaphase of the second maturation mitotis.

(fig. 6, X). At times, however, the $X$ chromosome apparently breaks away from its attachment to the large chromosome and divides alone in the second mitosis, forming two small, nearly spherical chromatin masses one of which goes to each pole of the spindle (fig. 5).

Since the $X$ chromosome passes undivided to one pole of the first maturation. spindle and divides longitudinally in the second maturation mitosis two classes of spermatozoa are produced in Necturus, as in many other species of animals. Presumably both classes of spermatozoa are functional, as I have not found sufficient degeneration among the spermatids to warrant the assumption 
that spermatocytes lacking the $X$ chromosome degenerate, as is the case in Phylloxerans (Morgan '09) and several other forms.

The mature spermatocytes of Necturus show a condition of the $X$ chromosome which is transitional between that found in the many species in which the $X$ chromosome can be traced as a separate structure throughout the development of the spermatocytes and the chromatin relations in. such forms as the higher batrachians in. which the $X$ chromosome has apparently formed a permanent union with one of the large chromosomes and can no longer be distinguished by any method of technique at our command. It is hoped that the study of the early stages in the development of the spermatocytes will give further facts of interest regarding the relations of the $X$ chromosome in this amphibian.

\section{LITERATURE CITED}

Carnoy, J. B. et Lebrun, H. 1899 La vésicule germinative et les globules polaires chez les batraciens. La Cellule, t. 16.

King, HeLen Dean 1905 The formation of the first polar spindle in the eggs of Bufo lentiginosus. Biol. Bull., vol. 9.

Ljebrun, H. 1901 La vésicule germinative et les globules polaires chez les batraciens. La Cellule, t. 20.

McClung, C. E. 1905 The chromosome complex of orthopteran spermatocytes. Biol. Bull., vol. 9.

Morgan, T. H. 1909 A biological and cytological study of sex determination in phylloxerans and aphids. Jour. Exp. Zoöl., vol. 7.

Stevens, N. M. 1912 a Supernumerary chromosomes and synapsis in Ceutophilus (sp. ?). Biol. Bull., vol. 22.

$1912 \mathrm{~b}$ Further observations on supernumerary chromosomes, and sex ratios in Diobrotica soror. Biol. Bull., vol. 22.

WIIson, E. B. 1909 Studies on chromosomes. V. The chromosomes of Metapodius. A contribution to the hypothesis of genetic continuity of chromosomes. Jour. Exp. Zoöl., vol. 6. 